\title{
Modelo Didáctico para el aprendizaje del Taller de Diseño Arquitectónico de una universidad de Trujillo - Perú
}

\author{
Didactic Model for the Learning of the Architectural Design Workshop \\ of a University in Trujillo - Peru \\ Modelo Didatico para a Aprendizagem da Oficina de Desenho Arquitectónico \\ de uma Universidade em Trujillo - Peru \\ LUIS ENRIQUE TARMA CARLO ${ }^{1}$ \\ KAREN PESANTES ALDANA ${ }^{2}$
}

\begin{abstract}
RESUMEN
La enseñanza del taller de diseño se basa en un aprendizaje práctico, orientado por los arquitectos con experiencia profesional, el propósito de la investigación, es demostrar si el modelo didáctico influye en el aprendizaje del Taller de diseño arquitectónico de una Universidad de Trujillo. El estudio es pre-experimental de tipo de investigación cuantitativa explicativa, el instrumento fue empleado en clase basado en la pedagogía del curso taller en la cual se elaboró una escala valorativa para sustentar la estructura teórica de la clase y que sirva de medio de comunicación entre docente y alumno. La población es de ciento dos alumnos cuya muestra es tomada en una sola sección de treinta y dos alumnos, se aplicó antes y después del taller. Concluyendo que el modelo didáctico si influye significativamente en el aprendizaje del taller de diseño arquitectónico.
\end{abstract}

Palabras clave: modelo didáctico; estilo de aprendizaje; arquitectura, taller de diseño.

\begin{abstract}
The teaching of the design workshop is based on practical learning, guided by architects with professional experience. The purpose of the research is to demonstrate if the didactic model influences the learning of the Architectural Design Workshop of a University of Trujillo. The study is a pre-experimental type of quantitative explanatory research, the instrument was used in class based on the pedagogy of the workshop course in which a scale was developed to support the theoretical structure of the class and to serve as a means of communication between teacher and student. The population is one hundred and two students whose sample is taken in a single section of thirty-two students, it was applied before and after the workshop. Concluding that the didactic model does influence significantly in the learning of the architectural design workshop.
\end{abstract}


Keywords: didactic model; learning style; architecture, design workshop.

\section{RESUMO}

O ensino da oficina de desenho baseia-se na aprendizagem prática, orientada por arquitetos com experiência profissional. O objetivo da investigação é demonstrar se o modelo didático influencia a aprendizagem da oficina de desenho arquitetônico de uma Universidade de Trujillo. O estudo é um tipo de pesquisa explicativa quantitativa pré-experimental, o instrumento foi utilizado nas aulas com base na pedagogia do curso de oficina, na qual foi desenvolvida uma escala para apoiar a estrutura teórica da aula e para servir como meio de comunicação entre professor e aluno. A população é de cento e dois alunos cuja amostra é recolhida numa única secção de trinta e dois alunos, foi aplicada antes e depois da oficina. Em conclusão, o modelo didático tem uma influência significativa no processo de aprendizagem da oficina de desenho arquitetônico.

Palavras-chave: modelo didático; estilo de aprendizagem; arquitetura, oficina de desenho.

\section{INTRODUCCIÓN}

Un aspecto tradicional es la enseñanza de carácter experimental y de aprendizaje práctico, orientado por consejos de un profesional en la carrera de Arquitectura, esto nace primero en la formación del arte y la cultura para luego extraerse a la formación del arquitecto, ello se evidencia en las facultades donde imparten artes de diseño en París bajo un modelo pedagógico centrado en el problema de diseño, que se iniciaba con un esquisse y terminando en charreitte (Delgado, 2007). La enseñanza moderna se focaliza en la Escuela de la Bauhaus (fundación: 1919, cese de operaciones: 1933) cuando Johannes Itten (1888-1967) pintor y pedagogo establecía que los criterios pedagógicos inicial en la formación del arquitecto se basaban en la "intuición y el método", es decir, en la capacidad de experimentar subjetivamente y de conocer objetivamente, para lo cual planteo trabajar con formas y colores básicos, para luego focalizar la formación en el proceso biológico "constructivo", no el proceso estético "diseño"; esto lleva a pensar que siempre fue una preocupación el cómo abordar la formación del arquitecto. Actualmente existen dos tendencias como lo establece Delgado (2007), un profesionalista que por su nombre "reproduce situaciones del ejercicio profesional" y la otra es buscar una "revaloración teórica de la práctica".

La definición de un "modelo didáctico" es de vital importancia en el aprendizaje del estudiante como la necesidad del nivel educativo de formación. Apoyándonos a construir la relación necesaria entre el análisis teórico y la práctica que son conexiones que muchas veces echamos de menos en el desarrollo de la práctica pedagógica que se haya tradicionalmente divididas por partes de elaboraciones teóricas que van asociadas de la multidisciplinariedad de teorías como en el campo de la sociología, psicología y, sobre todo, del campo curricular expresa también los materiales didácticos dentro del aula así como los grupos creativos que recién se innovan dentro del taller, estas actuaciones van dirigidas con el aporte de García \& Pérez (2000). 
Ayora Talavera \& Urzais Lares (1997) aborda la problemática de formación de los estudiantes de arquitectura desde un enfoque de enseñanza de diseño bidimensional y no de un diseño tridimensional este último necesario para el arquitecto y el fomento de la sensibilidad y capacidad de percepción de los estudiantes, tanto en los primeros niveles como al final, por lo que su muestra está referida a los dos talleres básicos I y II y a los últimos talleres arquitectónicos llamado taller terminal.

En ese sentido, los aprendizajes son parte esencial del conocimiento para llegar a entender cómo enseñar, es una relación muy importante en el binomio docente - alumno, para todo esto es imposible tener una sola teoría porque cada individuo tiene múltiples formas de aprender, de acuerdo a la variedad de aspectos afectivos y/o de factores psicológicos, además cognitivos, con participación en el desarrollo (Brookfield, 1995), además las inteligencias múltiples de Gardner (1985), que considera que el ser humano tiene un potencial biopsicológico necesario para demostrar habilidades diversas, además esto lo desarrolla o activa en un contexto cultural, para la resolución de problema o productos, en el caso del arquitecto establece que una de las inteligencias que mayor lo desarrolla es la Inteligencia espacial (manejar el espacio, distancia, tiempo y contexto).

Felder, (1996) establece que los alumnos abordan las formas de organizar y las maneras de procesar y asimilar la información y convertir en experiencia útil sobre un esquema proyectual. De acuerdo con Mabardi, (2006) es el alumno quien determina libremente las reglas y se somete a la formación, por tanto, se convierte en actor y motor principal de su formación y el docente como tutor. Como afirma Morín (2000), el docente debe construir el aprendizaje al alumno con la óptica de un pensamiento complejo que constituye el medio para construir el saber humano desde una visión hermenéutico que interprete la realidad y comprenda. Estas teorías dan relevancia a la variable independiente del Modelo didáctico, el cual, según Nuthall \& Snook (1973) es un esquema articulador entre la realidad y el pensamiento, basado en teorías y su desarrollo en su realización de la práctica. Es un esquema interpretativo que se selecciona las partes importantes de la realidad y los organiza de acuerdo a su jerarquía, según sus dimensiones. Según Entwistle (1987) el modelo heurístico enfatiza los tres elementos al interior del dinamismo docente, conformado por: alumno: con sus estrategia y formas de aprendizaje, motivacionales; Docente: con su modelo de enseñanza y sus características particulares; el espacio académico; Contexto: una atmósfera social particular que ayuda a definir la enseñanza y la evaluación del rendimiento.

El problema de la presente investigación se formula a partir la conocer, ¿de qué manera el modelo didáctico influye en el aprendizaje del Taller de diseño arquitectónico en los estudiantes de Arquitectura de una universidad de Trujillo - Perú? Como hipótesis se plantea que el modelo didáctico influye significativamente en el aprendizaje del Taller de diseño arquitectónico en los estudiantes de Arquitectura de una universidad de Trujillo - Perú.

El objetivo general de este informe es demostrar si el modelo didáctico influye en el aprendizaje de la asignatura Taller de diseño arquitectónico nueve en los estudiantes del noveno ciclo de Arquitectura de una universidad, Trujillo. Como objetivos específicos se propone, identificar el nivel de aprendizaje antes y después del Modelo Didáctico aplicado en estudiantes de la asignatura Taller de Diseño Arquitectónico; diseñar el modelo didáctico y su aplicación antes y después según dimensiones de los aprendizajes en la asignatura taller de diseño arquitectónico en los estudiantes de arquitectura; identificar el nivel influencia 
en las dimensiones de aprendizaje de la asignatura taller de diseño arquitectónico en los estudiantes, antes y después de aplicar el modelo didáctico; y medir la influencia del modelo didáctico en el aprendizaje en la asignatura taller de diseño arquitectónico en los estudiantes de arquitectura.

\section{METODOLOGÍA}

La presente investigación es de tipo preexperimental de tipo de investigación: cuantitativa explicativa que permite realizar la medición previa de la variable dependiente a ser estudiada (pre test), la aplicación de la variable independiente o experimental $\mathrm{X}$ con el sujeto $\mathrm{Y}$, así como la medición nueva de la variable dependiente con el sujeto (post test). (Hernández et al., 2010).

En análisis de los datos de la investigación se realizó mediante la estadística descriptiva, utilizándose tablas de distribución de frecuencias absolutas simples y porcentuales para describir los niveles de cada variable. De la estadística inferencial se aplicó la prueba de Normalidad de Shapiro Wilk, identificándose distribuciones diferentes a la distribución normal en la dimensión análisis y evaluación de proyectos y una distribución normal en las dimensiones esquematización, problematización y en el aprendizaje, decidiéndose utilizar la prueba no paramétrica de Wilcoxon para realizar la comparación en la dimensión análisis y evaluación de proyectos y la prueba t-Student para las dimensiones esquematización y problematización en el Aprendizaje.

\section{RESULTADOS}

Analizando los resultados por objetivos, en cuanto a identificar el nivel de aprendizaje de la asignatura Taller de Diseño Arquitectónico en los estudiantes de arquitectura, que establece y muestra un aprendizaje de nivel medio en un $69 \%$ en el pre-test y de nivel alto en un $94 \%$ en el post-test en estudiantes de la asignatura Taller de Diseño Arquitectónico de una Universidad de Trujillo - Perú.

Respecto al objetivo de diseñar el modelo didáctico y su aplicación en la asignatura taller de diseño arquitectónico en los estudiantes de arquitectura, la aplicación del modelo didáctico antes y después, según la dimensión Esquematización representa un nivel medio en un 63\% en el pre-test y de nivel alto en un $88 \%$ en el post-test. Así mismo para la dimensión Problematización representa un nivel medio en un $63 \%$ en el pre-test y de nivel alto en un $88 \%$ en el post-test, de igual manera para la dimensión análisis y evaluación con un nivel medio en $69 \%$ en el pre-test y de nivel alto en un $94 \%$ en el post-test en estudiantes del Taller de Diseño Arquitectónico Nueve de una Universidad de Trujillo - Perú.

Con relación al objetivo identificar el nivel de aprendizaje de la asignatura taller de diseño arquitectónico en los estudiantes, después de aplicar el modelo didáctico, mediante el posttest y pre-test, se aprecia una media de 13.44 (5.94) en el pre-test y de 28.56 (3.41) en el post-test, haciendo una diferencia pareada de 15.13 (6.29), señalando una influencia estadísticamente significativa $(\mathrm{p}<.05)$ del Modelo didáctico en la Esquematización, además de una media de 14.63 (4.87) en el pre-test y de 27.75 (2.70) en el post-test, haciendo una 
diferencia pareada de 13.13 (5.84), señalando una influencia estadísticamente significativa $(\mathrm{p}<.05)$ del Modelo didáctico en la Problematización y un rango promedio de 8.5 en los rangos negativos, denotando rangos menores en el pre-test respecto del post-test, evidenciando una influencia estadísticamente significativa $(\mathrm{p}<.05)$ del Modelo didáctico en Análisis y Evaluación de Proyectos en estudiantes del Taller de Diseño Arquitectónico Nueve en una Universidad de Trujillo, 2018.

En cuanto al objetivo medir los resultados del aprendizaje en la asignatura taller de diseño arquitectónico en los estudiantes de Arquitectura. se aprecia una media de 32.38 (10.0) en el pre-test y de 66.25 (6.52) en el post-test, haciendo una diferencia pareada de 33.88 (12.04), señalando una influencia estadísticamente significativa $(\mathrm{p}<.05)$ del Modelo didáctico en el Aprendizaje en estudiantes del Taller de Diseño Arquitectónico Nueve en una Universidad de Trujillo - Perú.

\section{DISCUSIÓN}

La estrategia didáctica proyectual que debe impartir el docente se basa en la formación construida donde los alumnos son actores y motores principales de su formación y el docente como tutor (Mabardi, 2006). Además, es necesario conocer y entender las formas de aprender para que el docente tenga respuesta o estrategia didáctica efectivas, esto permitirá lograr lo planificado en la sumilla y competencia del alumnos; requiriendo establecer la necesidad que el aprendizaje tiene que ver con las dimensiones de esquematizar (organizar la información), problematizar los saberes (procesar la información), para luego analizar y evaluar la respuesta proyectual (convertir la información en experiencia proyectual), esto tiene que ver como el alumno abordar las formas de organizar y la maneras de procesar y asimilar la información y convertir en experiencia útil (Felder, 1996).

El resultado muestra que el Aprendizaje antes y después de la aplicación del Modelo Didáctico tiene un nivel medio en un 69\% en el pre-test y de nivel alto en un $94 \%$ en el post-test, determinando que el modelo didáctico, permite interiorizar en el alumno para acomodar la información, procesar y a partir de ello construir su propio conocimiento, debido a la secuencia didáctica y a la construcción del aprendizaje es decir de interpretar la realidad y comprenderla, lograr explicarla, cuantificarla y objetivarla (Morín, 2000), pero todo eso se da siempre el saber se construye haciendo, como expresa Machado (1998) "Caminante, no hay camino se hace camino al andar", por lo que el aprendizaje en el taller de diseño arquitectónico se debe construir mediante un proceso creativo y complejo considerando que este nivel no solo se entrelaza la especialidad si no otras ciencias (sociales, económicas, culturales, ambientales y especialidades afines a la carrera) para el diseño y producción del conocimiento proyectual.

La aplicación del modelo didáctico antes y después, según la dimensión Esquematización representa un nivel medio en un $63 \%$ en el pre-test y de nivel alto en un $88 \%$ en el posttest. Asimismo, la dimensión Problematización representa un nivel medio en un $63 \%$ en el pre-test y de nivel alto en un $88 \%$ en el post-test, de igual manera para la dimensión análisis y evaluación con un nivel medio en $69 \%$ en el pre-test y de nivel alto en un 94\% en el post-test en estudiantes del Taller de Diseño Arquitectónico Nueve de una Universidad de Trujillo - Perú, por lo que el modelo, representan una diferencia importantes en los 
aprendizajes de los alumnos. Esto nos permite establecer que el aprendizaje en el taller de diseño, el alumno mismo lo construye, desde un enfoque de vida humana (actitud y aptitud lograda) y la relación social (percepción, lugar, objeto, sujeto), para procesar y entender el conocimiento del proceso y la producción proyectual, esto se explica, que la construcción del conocimiento se entrelaza entre el hombre, la sociedad, la vida y el mundo (Rozo, 2003, citado por Tobón, Sergio 2005), por lo que el aprendizaje de los alumnos de la asignatura se basa en identificar las necesidades en una realidad (social, cultural, económica o ambiental) en un lugar (rural urbano con o sin compromiso) y factualidad (gestión de la necesidad y el objeto a proyectar y construir), como requisitos básicos para lograr una competencia basado en modelo heurístico que el docente incorpora como estrategia didáctica para encadenar e incentivar el descubrimiento interno individual de alumnos en beneficio propio.

Si analizamos la Influencia del Modelo didáctico en la Esquematización en el pre-test y post-test, se tiene una diferencia pareada de 15.13 (6.29), señalando una influencia estadísticamente significativa $(\mathrm{p}<.05)$, además la Influencia del Modelo didáctico en la Problematización durante el pre-test y post-test, hace una diferencia pareada de 13.13 (5.84), señalando una influencia estadísticamente significativa $(\mathrm{p}<.05)$. De igual manera, la Influencia del Modelo didáctico en el Análisis y Evaluación de Proyectos, representa rango promedio de 8.5 en los rangos negativos, denotando rangos menores en el pre-test respecto del post-test; ello evidencia una influencia estadísticamente significativa $(\mathrm{p}<.05)$; que se concatena con los aprendizajes logrados en el taller de diseño arquitectónico, saber esquematizar el trabajo mediante un esquema metodológico proyectual (recolección de la información basados en los elementos del proceso de diseño: contexto, objeto, usuario), problematización (contextualizar y procesar la información mediante los elementos del proceso de diseño: contexto, objeto, usuario identificando las causas y medios) y el análisis y evaluación de la propuesta del proyecto (mediante las conclusiones cualitativas y cuantitativas: terreno, programa arquitectónico y partido arquitectónico o variables de diseño), logrado mediante la covaloración que es una estrategia en la cual los alumnos valoran entre ellos sus capacidades de acuerdo a sus criterios previos, determinados y definidos, por lo que el estudiante recibe un proceso de retroalimentación de sus pares respecto a su aprendizaje y logro Tobón (2005), representa al docente como un tutor y acompañante, esta forma de evaluación permite al alumno, prepararse para aceptar y resolver los problemas y las críticas en un contexto profesional.

En general si se aprecia la importancia del modelo didáctico en el aprendizaje de los alumnos, esto representa una influencia significativa en el aprendizaje en estudiantes del taller de diseño arquitectónico nueve, entre el pre test y post test, se tiene una diferencia pareada de 33.88 (12.04), señalando una influencia estadísticamente significativa $(p<.05)$ del modelo didáctico en el aprendizaje por lo que las dimensiones nos ha permitido establecer que con el modelo didáctico heurístico, los aprendizajes logrados son significativos, esto debido a la característica del método, que los trabajos finales del proyecto Arquitectónico lo realizan en grupo mínimo de dos a cuatro, determinando que el modelo didáctico Heurístico desarrolla lo participativo, colaborativo y trabajo en equipo, esto quiere decir que el modelo permite crear, procesos de pensamiento, en la cual el alumno se convierte en un pensador activo. El pensador activo se define por que reordena de varias maneras la información de un problema antes de alcanzar a una comprobada solución (Parra, 2001). 
En general, la influencia del modelo Didáctico en el aprendizaje de los alumnos en el Taller de diseño arquitectónico del noveno ciclo constituye un importante conocimiento para lograr el vínculo y armonía entre el alumno y el docente. Según Entwistle (1987) este modelo enfatiza en los tres elementos al interior del dinamismo docente, conformado por: el alumno con sus estrategia y estilos o formas de aprendizaje, faces de personalidad y los mecanismos motivacionales; el docente con su modelo de enseñanza y sus características particulares; el espacio académico (taller de diseño arquitectónico), con su propio perfil del quehacer disciplinario (proyecto arquitectónico), una atmosfera social particular que ayuda a definir las enseñanza y la evaluación del rendimiento.

\section{CONCLUSIONES}

El modelo didáctico sí permite el logro del aprendizaje de los estudiantes del Taller de Diseño Arquitectónico nueve en una facultad de Arquitectura, debido a que el Nivel de Aprendizaje antes y después del Modelo Didáctico; muestra un aprendizaje de nivel medio en un $69 \%$ en el pre-test y de nivel alto en un $94 \%$ en el post-test en estudiantes del Taller de Diseño Arquitectónico Nueve de una Universidad de Trujillo, 2018.

La evaluación del aprendizaje del Taller de Diseño en los estudiantes de arquitectura del décimo ciclo de una Universidad de Trujillo - Perú, en el Nivel de Esquematización antes y después del Modelo Didáctico representa un nivel medio en un $63 \%$ en el pre-test y de nivel alto en un $88 \%$ en el post-test, además la problematización tiene un nivel medio en un $63 \%$ en el pre-test y nivel alto en un $88 \%$ en el post-test, también el análisis y evaluación de nivel medio en un $69 \%$ en el pre-test y de nivel alto en un $94 \%$ en el post-test en estudiantes del Taller de Diseño Arquitectónico Nueve de una Universidad de Trujillo.

Las dimensiones del Aprendizaje de los alumnos del Taller de Diseño Arquitectónico nueve, sí representa una influencia significativa cuando se aplica el modelo didáctico por lo que los resultados evidencian que la diferencia entre el pre test y el post-test, establece una diferencia pareada de 15.13 (6.29), para la esquematización, una diferencia pareada de 13.13 (5.84), para la Problematización, y rango promedio de 8.5 en los rangos negativos, denotando rangos menores en el pre-test respecto del post-test, evidenciando una influencia estadísticamente significativa $(\mathrm{p}<.05)$ en el Análisis y Evaluación de Proyectos, que si existe influencia de la carrera de Arquitectura en el Aprendizaje del Taller de Diseño Arquitectónico Nueve en una Universidad de Trujillo, 2018.

El modelo didáctico sí tiene influencia en el Aprendizajes de los alumnos del Taller de Diseño Arquitectónico Nueve, porque se aprecia una media de 32.38 (10.0) en el pre-test y de 66.25 (6.52) en el post-test, haciendo una diferencia pareada de 33.88 (12.04), señalando una influencia estadísticamente significativa $(\mathrm{p}<.05)$ del Modelo didáctico en el Aprendizaje en estudiantes del Taller de Diseño Arquitectónico Nueve en una Universidad de Trujillo, 2018. 


\section{REFERENCIAS}

Ayora Talavera, S., \& Urzais Lares, E. (1997). Enseñanza-aprendizaje del diseño arquitectónico. https://pdfs.semanticscholar.org/7ede/abdd2b99c75db91c0e99fe 747c4b7b299451.pdf

Brookfield, S. (1995). The getting of wisdom: What critically reflective teaching is and why it's important. Becoming a critically reflective teacher, 1-28. https://mrug. pw/1594254638.pdf

Entwistle, N. (1987). Entender el aprendizaje en el aula. Hodder y Stoughton.

Felder, R. M. (1996). Cuestiones de Estilos: Principios y aplicaciones de cuatro modelos de estilo de aprendizaje. ASEE Prism.

Gardner, H. (1995). Mentes creativas. Una anatomía de la creatividad vista a través de las vidas de S. Freud, A. Einstein, P. Picasso, I. Stravincky, T.S. Elliot, M. Graham, M. Gandhi. Paidos.

Hernández, R., Fernández, C., \& Baptista, M. (2010). Metodología de la Investigación. McGraw Hill. https://www.esup.edu.pe/descargas/dep_investigacion/Metodologia\%20 de\%201a\%20investigaci\%C3\%B3n\%205ta\%20Edici\%C3\%B3n.pdf

Mabardi, F. (2006). Conferencia sobre Enseñanza en la Arquitectura. Pontificia Universidad Católica del Perú, Lima.

Machado, A. (1998). Caminante. Planeta.

Morín, E. (2000). Los siete saberes necesarios para la educación del futuro. Ministerio de Educación Nacional.

Nuthall, G., \& Snook, I. (1973). Contemporary models of teaching. Second handbook of research on teaching. Ran McNally.

Parra, M. (2001). Salud mental y trabajo. Monografias de gestión en psiquiatría y salud mental. Universidad de Santiago de Chile.

Tobón, S. (2005). Formación basada en competencias: Pensamiento complejo, diseño curricular y didáctica. Ecoe ediciones. http://atlas.umss.edu.bo:8080/xmlui/handle/123456789/1152 